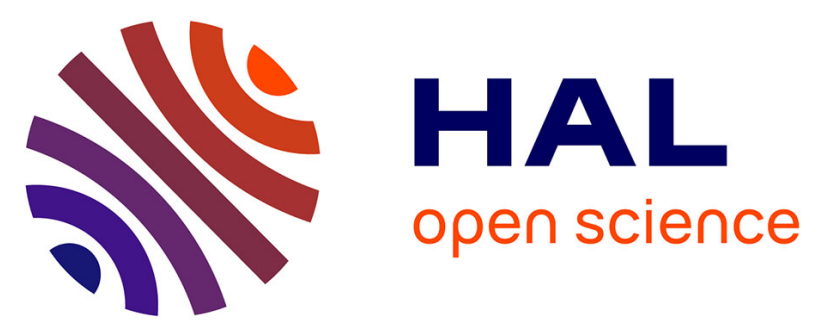

\title{
Electron-Phonon Scattering in Metal Clusters
}

Arnaud Arbouet, C. Voisin, D. Christofilos, Pierre Langot, N. del Fatti, F.

Vallée, J. Lerme, G. Celep, E. Cottancin, M. Gaudry, et al.

\section{To cite this version:}

Arnaud Arbouet, C. Voisin, D. Christofilos, Pierre Langot, N. del Fatti, et al.. Electron-Phonon Scattering in Metal Clusters. Physical Review Letters, 2003, 90 (17), pp.177401(1-4). 10.1103/PhysRevLett.90.177401 . hal-00342480

\section{HAL Id: hal-00342480 https://hal.science/hal-00342480}

Submitted on 23 Apr 2018

HAL is a multi-disciplinary open access archive for the deposit and dissemination of scientific research documents, whether they are published or not. The documents may come from teaching and research institutions in France or abroad, or from public or private research centers.
L'archive ouverte pluridisciplinaire HAL, est destinée au dépôt et à la diffusion de documents scientifiques de niveau recherche, publiés ou non, émanant des établissements d'enseignement et de recherche français ou étrangers, des laboratoires publics ou privés. 


\title{
Electron-Phonon Scattering in Metal Clusters
}

\author{
A. Arbouet, C. Voisin, ${ }^{*}$ D. Christofilos, P. Langot, N. Del Fatti, and F. Vallée \\ Centre de Physique Moléculaire Optique et Hertzienne, CNRS and Université Bordeaux I, 351 cours de la Libération, \\ 33405 Talence, France \\ J. Lermé, G. Celep, E. Cottancin, M. Gaudry, M. Pellarin, and M. Broyer \\ Laboratoire de Spectrométrie Ionique et Moléculaire, CNRS and Université Lyon I, 43 Boulevard du 11 Novembre 1918, \\ 69622 Villeurbanne, France \\ M. Maillard and M. P. Pileni \\ Laboratoire des Matériaux Mésoscopiques et Nanométriques, CNRS and Université Pierre et Marie Curie, 4 place Jussieu, \\ 75252 Paris cedex 05, France \\ M. Treguer \\ Institut de Chimie de la Matière Condensée de Bordeaux, CNRS and Université Bordeaux I, 87 Avenue du Dr. Albert Schweitzer, \\ 33608 Pessac, France
}

(Received 6 December 2002; published 29 April 2003)

\begin{abstract}
Electron-lattice energy exchanges are investigated in gold and silver nanoparticles with sizes ranging from 30 to $2.2 \mathrm{~nm}$ embedded in different environments. Femtosecond pump-probe experiments performed in the low-perturbation regime demonstrate a strong increase of the intrinsic electronphonon interaction for nanoparticles smaller than $10 \mathrm{~nm}$ due to a confinement effect.
\end{abstract}

PACS numbers: 78.67.Bf, 42.65.-k, 78.47.+p

The interactions of electrons between themselves and with their environment are key effects in the fundamental properties of condensed matter. Their modifications close to a surface or by confinement in reduced dimensionality systems are of central interest for the understanding of the new properties of these materials. Time-resolved femtosecond techniques have been shown to be powerful tools for the investigation of the different electron scattering processes. In the case of metals, they have been extensively used in bulk systems and have been recently extended to metal clusters [1,2], yielding, for instance, information on the intrinsic electron-electron coupling [3]. The impact of confinement on the cooling of hot electrons, i.e., on energy redistribution to their environment, has also been addressed, with, however, contradictory results on the size effects. No dependence, large variations, or even a nonmonotonic behavior have been reported in the one to few tens nanometer size range $[1,2,4-7]$. As most of the experiments were performed in the strong excitation regime, these discrepancies can be ascribed to the pump intensity dependence of the measured kinetics [see Eq. (1) below] and to the large influence of the surrounding material $[2,6,8-10]$. This has precluded unambiguous determination of the intrinsic electron-lattice interaction in metal nanoparticles and of its modification by confinement, processes that, to our knowledge, have not yet been investigated by other techniques.

We have performed a systematic investigation of the electron-lattice energy exchanges in silver and gold nanoparticles in the weak excitation regime (induced maxi- mum electron temperature rise $\Delta T_{e}^{m}$ smaller than 100$200 \mathrm{~K}$ [11]) using a high sensitivity femtosecond absorption saturation technique. The results were found to be independent of the nanoparticle environment and growing method. They yield evidence for an increase of the intrinsic electron-lattice interactions in metal clusters with sizes smaller than $10 \mathrm{~nm}$.

Our experimental technique is an extension of that used in noble metal films [12,13]. It relies on the close connection between the energy distribution of the conduction electrons and the optical response, i.e., the metal dielectric function. This can be separated into an intraconduction band (free electrons) contribution and an interband one, related to $d$ bands to conduction band transitions. The absorption threshold $\hbar \Omega_{\mathrm{ib}}$ for these transitions is in the ultraviolet-visible range, with $\hbar \Omega_{\mathrm{ib}} \approx$ $4 \mathrm{eV}$ in $\mathrm{Ag}$ and $2.3 \mathrm{eV}$ in Au. In time-resolved experiments, energy is first selectively injected in the conduction electrons by a femtosecond pump pulse. The different steps of their relaxation, i.e., internal thermalization of the electron gas and its cooling, are followed by monitoring the transient optical response of the sample (transmission $\Delta T$ or reflection $\Delta R$ change) using a timedelayed probe pulse. It has been shown that, for a probe photon energy well below $\hbar \Omega_{\mathrm{ib}}$, the signal amplitude is only weakly sensitive to the electron distribution and is almost proportional to the electron gas excess energy $\Delta u_{e}\left(t_{D}\right)$ (defined as the difference between the electron gas energy at time $t_{D}$ and the one before perturbation, $t_{D}$ being the pump-probe delay) $[13,14]$. For this off-resonant probing condition, the time evolution of 
the measured signal thus directly reflects the energy losses of the electrons to their environment, permitting its precise analysis.

In thin metal films this is limited to the lattice. After its internal thermalization at temperature $T_{e}$, the cooling dynamics of the electron gas and its thermalization with the lattice can be described by the two-temperature model [15]:

$$
\begin{aligned}
C_{e}\left(T_{e}\right)\left(\partial T_{e} / \partial t\right) & =-G\left(T_{e}-T_{L}\right), \\
C_{L}\left(\partial T_{L} / \partial t\right) & =G\left(T_{e}-T_{L}\right),
\end{aligned}
$$

where $T_{L}$ is the lattice temperature and $G$ is the electronphonon coupling constant. For a weak perturbation $\Delta T_{e}^{m e} \ll T_{0}$, where $T_{0}$ is the initial temperature, the electron heat capacity $C_{e}\left(T_{e}\right)$ can be identified with $C_{e}\left(T_{0}\right)$. An exponential decay of the electron temperature rise $\Delta T_{e}$ and of $\Delta u_{e}$, with the time constant $\tau_{e-\mathrm{ph}}^{0} \approx$ $C_{e}\left(T_{0}\right) / G$ is predicted, permitting direct determination of $G$ [12-14]. It is not the case for a strong excitation where, as $C_{e}$ rises with $T_{e}$, the $\Delta T_{e}$ decay is slowed down and becomes nonexponential and excitation dependent, making difficult precise extraction of $G$. A similar approach can be used in noble metal nanoparticles, taking into account that for embedded particles the electron energy can also be transferred to the surrounding material, possibly modifying the observed kinetics as observed for large excitation $[6,8-10]$.

The experiments were performed in samples formed by spherical silver or gold nanoparticles embedded either in a $50 \mathrm{BaO}-50 \mathrm{P}_{2} \mathrm{O}_{5}, \mathrm{Al}_{2} \mathrm{O}_{3}, \mathrm{MgF}_{2}$, or polymer [polymethylmethacrylate (PMMA)] matrix, in aqueous solution or supported on glass. The average nanoparticle diameter $D$ of each sample was measured by transmission electron microscopy and ranges from 30 to $3.2 \mathrm{~nm}$ for $\mathrm{Ag}$ and from 20 to $2.2 \mathrm{~nm}$ for $\mathrm{Au}$, with a size dispersion of the order or smaller than $10 \%$. The $50 \mathrm{BaO}-50 \mathrm{P}_{2} \mathrm{O}_{5}$ glass samples were prepared by a fusion and heat treatment technique [16]. The $\mathrm{Al}_{2} \mathrm{O}_{3}$ and $\mathrm{MgF}_{2}$ samples were grown using low energy cluster beam deposition (LECBD) with codeposition of the matrix [17]. The colloidal solutions were prepared by chemical synthesis using a reverse micelle (Ag) [18] or a radiolysis (Au) [19] technique. The thiol stabilized nanoparticles were left in solution, embedded in PMMA, or dispersed on a glass substrate.

The experimental setup is based on a femtosecond frequency tunable Ti:sapphire oscillator delivering $25 \mathrm{fs}$ near-infrared pulses. The pulse train is split in two parts. The first one is used to create the nonequilibrium electron distribution with a pump pulse at either the fundamental, $\hbar \omega_{\mathrm{pp}} \approx 1.45 \mathrm{eV}$, or doubled frequency, $\hbar \omega_{\mathrm{pp}} \approx 2.9 \mathrm{eV}$, of the laser. The second part, at the fundamental (for $\mathrm{Au}$ ) or doubled (for $\mathrm{Ag}$ ) laser frequency, is used to probe the induced sample transmission change $\Delta T / T$. The two beams were sent in a pump-probe setup with mechanical chopping of the pump beam and lock-in detection of the probe beam transmission change $\Delta T$ (defined as the difference between the perturbed and the unperturbed sample transmission). The high sensitivity of our apparatus with a noise level for $\Delta T / T$ in the $10^{-6}$ range permits measurements to be performed in the weak excitation regime with $\Delta T_{e}^{m}$ in the $10-200 \mathrm{~K}$ range.

The time evolution of $\Delta T / T$ measured in silver nanoparticle samples with $D=3,6$, and $26 \mathrm{~nm}$ are shown in Fig. 1. After a fast rise following energy injection, $\Delta T / T$ exhibits a size-dependent decay kinetics followed by a small amplitude background $(\Delta T / T)_{b}$ for long time delays. This residual signal corresponds to an electronlattice quasiequilibrium in each nanoparticle at a temperature larger than $T_{0}$ and decays with heat diffusion in the surrounding medium. The size dependence of the signal relaxation is more clearly shown by plotting $\Delta T / T$ on a logarithmic scale after subtracting $(\Delta T / T)_{b}$ (inset of Fig. 1). As expected for weak excitation regime studies, an exponential decay is observed, permitting one to determine the electron energy loss time. It is found to strongly decrease with size from about 850 fs for $D=$ $26 \mathrm{~nm}$ to about 500 fs for $D=3 \mathrm{~nm}$ in $\mathrm{Ag}$, with similar results in gold, yielding evidence for faster electron cooling in small particles (Fig. 2).

The measured decay times were checked to be independent of the probe wavelength and of both the pump fluence and the pump photon energy (using a pump pulse at either the fundamental or the second harmonic of the laser). We emphasize that the minimum perturbation that can be induced in each excited particle is set by absorption of one pump photon, which corresponds to our situation for the smallest investigated clusters. Independence of the time response on both parameters is thus necessary to characterize the weak perturbation

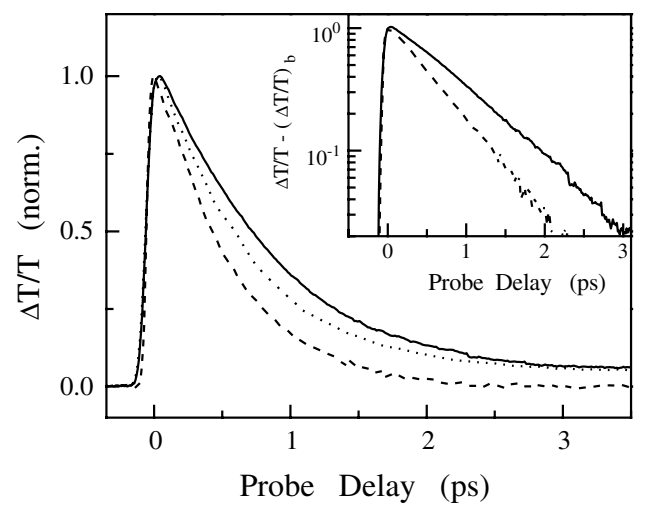

FIG. 1. Time dependence of the normalized transmission change $\Delta T / T$ measured in spherical $\mathrm{Ag}$ clusters of mean diameter $D=26$ and $6 \mathrm{~nm}$ in $\mathrm{BaO}-\mathrm{P}_{2} \mathrm{O}_{5}$ (solid and dotted lines) and $D=3 \mathrm{~nm}$ in $\mathrm{Al}_{2} \mathrm{O}_{3}$ (dashed line) for pump and probe photon energies $\hbar \omega_{\mathrm{pp}} \approx 1.48 \mathrm{eV}$ and $\hbar \omega_{\mathrm{pr}} \approx 2.96 \mathrm{eV}$. The inset shows the $D=26$ and $3 \mathrm{~nm}$ data on a logarithmic scale after subtraction of the long delay signal $(\Delta T / T)_{b}$. The decay times are 850 and $530 \mathrm{fs}$, respectively. 


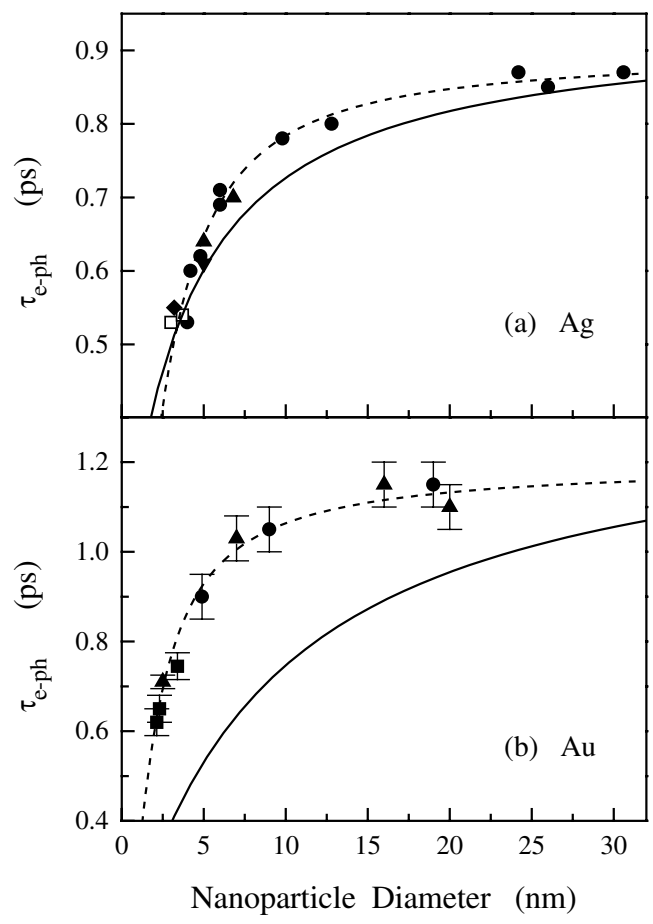

FIG. 2. (a) Size dependence of the measured electron-lattice energy exchange time $\tau_{e \text {-ph }}$ for silver clusters in $\mathrm{BaO}-\mathrm{P}_{2} \mathrm{O}_{5}$ (dots), $\mathrm{Al}_{2} \mathrm{O}_{3}$ (open squares), $\mathrm{MgF}_{2}$ (diamond), a polymer (up triangles), or deposited on a glass substrate (down triangle). The solid line is the computed $\tau_{e \text {-ph }}$ taking into account the spillout and $d$-electron localization effects, normalized to its bulk value for large particles. The dashed line is a guide to the eye. (b) Same as (a) for gold clusters in $\mathrm{Al}_{2} \mathrm{O}_{3}$ (squares) or in colloidal solutions prepared using a radiolysis technique (triangles) or commercially available (dots).

regime, since below a certain fluence only the number of excited particles is reduced, not their excitation level [2]. This demonstrates that our measurements were performed in equivalent conditions in the different samples and that the measured electron energy loss times can be directly compared.

Conversely to the experiments performed in the strong excitation regime, no influence of the nanoparticle environment nor of the preparation method has been observed (Fig. 2). This is illustrated in Fig. 3 for nanoparticles in the $3 \mathrm{~nm}$ size range embedded in a solid $\left(\mathrm{Al}_{2} \mathrm{O}_{3}\right)$ or in colloidal solution. The measured decay can thus be ascribed to the electron energy losses inside each nanoparticle, i.e., to the intrinsic electron-lattice energy exchanges. In both metals, the times measured in large nanoparticles are very close to the ones determined in $25 \mathrm{~nm}$ thick films in the same experimental conditions (Fig. 2). As usually done, assuming that the latter can be identified with the bulk material coupling time $\tau_{e-\mathrm{ph}}^{0}$, our results show that clusters with sizes larger than about $10 \mathrm{~nm}$ have a bulklike behavior. A strong reduction of the decay time is observed for smaller sizes yielding

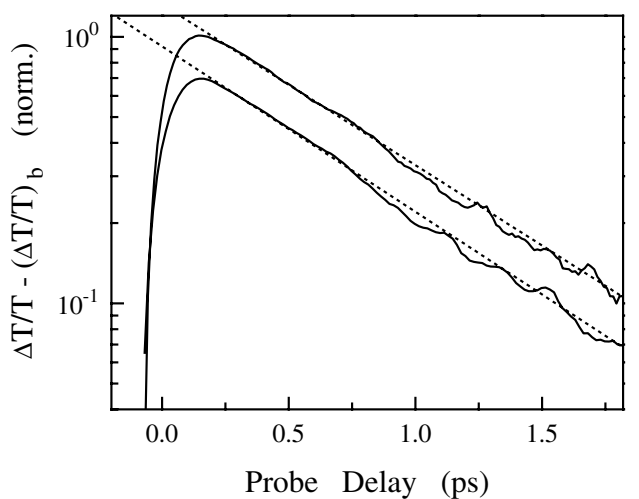

FIG. 3. Temporal evolution of the normalized transmission change $\Delta T / T-(\Delta T / T)_{b}$ measured in gold nanoparticles with mean diameter $D=2.5 \mathrm{~nm}$ in solution (lower curve) and $D=$ $3.4 \mathrm{~nm}$ in a $\mathrm{Al}_{2} \mathrm{O}_{3}$ matrix (upper curve). The samples were prepared using chemical or vapor phase (LECBD) synthesis, respectively. The dotted lines correspond to exponential decays with $\tau_{e \text {-ph }}=710$ and $730 \mathrm{fs}$, respectively. The pump and probe photon energies are $\hbar \omega_{\mathrm{pp}} \approx 2.9 \mathrm{eV}$ and $\hbar \omega_{\mathrm{pr}} \approx 1.45 \mathrm{eV}$.

evidence for a confinement-induced increase of the electron-lattice interaction in small metal nanoparticles.

The size behavior of the electron cooling kinetics is qualitatively similar to the one of electron-electron energy exchange measured previously [3]. The latter has been interpreted in terms of reduction of the screening of the electron-electron scattering close to the cluster surface, an effect which is also expected to modify the electron-ion interaction. We have estimated its impact on the electron-lattice energy exchanges using a similar phenomenological model consisting in spatially averaging in a cluster the parameters governing the local screening efficiency, namely, the local electron density $n_{e}$ and the static interband dielectric constant $\epsilon_{d}(0)$. Using standard approximations [20], one can show that the dependence of the bulk material $e$-ph scattering rate on the two above-mentioned parameters is given by:

$$
\frac{1}{\tau_{e-\mathrm{ph}}^{0}} \propto G \propto \int_{0}^{q_{D}} q^{3}\left|\frac{V(q)}{\epsilon(0, q)}\right|^{2} d q,
$$

where $q$ is the phonon momentum and $q_{D}$ the Debye wave vector. $V(q)$ is the unscreened electron-ion interaction potential and $\epsilon(0, q)$ the static small momentum limit of the dielectric function:

$$
\epsilon(0, q)=\epsilon_{d}(0)\left[1+\frac{1}{\epsilon_{d}(0)}\left(\frac{\beta q_{\mathrm{TF}}}{q}\right)^{2}\right] .
$$

$q_{\mathrm{TF}} \propto n_{e}^{1 / 6}$ is the Thomas-Fermi wave vector and $\beta=$ 0.73 a phenomenological factor introduced to correct for screening overestimation using Eq. (3) [13].

In a cluster, the electron wave functions are modified by surface effects, leading to the well-known conduction electron spillout and $d$-electron localization in the cluster 
core [21-23]. These effects lead to a reduction of the local values of $n_{e}$ and $\epsilon_{d}$, respectively, and thus of the screening of the electron-ion interaction. Using the bulk material results for the $e$-ph coupling rate [Eqs. (2) and (3)] and spatially averaging it over the nanoparticle volume using the known parameters for the spillout and $d$-electron localization effects [21-23], a large increase of the average scattering rates is computed in small clusters (Fig. 2). Similar results were obtained for different electron-ion interaction potentials $V(q)$, i.e., Coulomb potential or empty-core Ashcroft pseudopotential. The calculated size dependence of $\tau_{e \text {-ph }}$ is in qualitative agreement with the measured one indicating an important contribution due to screening reduction effect (Fig. 2).

A much better reproduction of the data is obtained in silver than in gold, but is probably fortuitous. The stronger size dependence calculated in gold is due to the larger $d$-electron contribution to screening [with $\epsilon_{d}^{\mathrm{Au}}(0)=$ 6.7 and $\epsilon_{d}^{\mathrm{Ag}}(0)=3.7$ [13]]. However, it has to be noted that the dependence of the bulk $\tau_{e \text {-ph }}^{0}$ on $\epsilon_{d}(0)$ given by Eq. (2) does not permit one to reproduce its change from gold to silver (about 30\% experimentally as compared to a predicted factor of about 3 ). This overestimated dependence can be partly at the origin of the larger predicted than measured size dependence in gold.

In the above model, quantum size effect on the cluster vibrations has been neglected. It modifies the vibration modes density of states, especially close to the surface [24], and possibly influences the electron-lattice interaction. A correlated effect, with different $\mathrm{Ag}$ and Au surface phonon mode behaviors [25], has been evoked to analyze electron surface state lifetime at a metal surface [26], together with the previously evoked screening reduction effects [27]. A model of electron coupling with surface vibration states has been developed by Belotskii and Tomchuk [28]. It is limited to small clusters and does not permit one to predict the continuous evolution of the electron-lattice interaction from a bulk to a confined material. In the case of gold, it predicts a larger energy transfer time in small clusters than in the bulk [28], in contrast with our observations. However, this effect can partly counterbalance the overestimated $\tau_{e \text {-ph }}$ reduction with size calculated taking into account only reduced screening. We stress here that a more correct approach should introduce quantum size effects on both the electronic and vibrational states and interaction between them, but is out of the scope of this Letter.

In conclusion, we have performed a systematic investigation of nonequilibrium electron energy losses in metal nanoparticles using a time-resolved femtosecond technique in the low-perturbation regime. The results obtained in silver and gold nanoparticles demonstrate that the electron-lattice energy exchanges are comparable to that measured in metal films for large particles and strongly increase for smaller ones $(D \leq 10 \mathrm{~nm})$. This size behavior is comparable to that measured for electron-electron interaction and is in qualitative agreement with a similar model based on a bulk approach modified by surface effects. Further, more elaborated modeling, taking into account both the electron and phonon confinement effects, is necessary to quantitatively reproduce the experimental data.

The authors thank A. Nakamura for helpful discussions and $\mathrm{S}$. Omi for providing the $\mathrm{BaO}-\mathrm{P}_{2} \mathrm{O}_{5}$ embedded silver nanoparticles. A. A., D. C., P. L., N. D. F., and F.V. also acknowledge financial support by the Conseil Régional d'Aquitaine.

*Present address: Laboratoire de Physique de la Matière Condensée, Ecole Normale Supérieure, 24 rue Lhomond, 75005 Paris, France.

[1] S. Link and M. A. El-Sayed, J. Phys. Chem. B 103, 8410 (1999), and references therein.

[2] C. Voisin, N. Del Fatti, D. Christofilos, and F. Vallée, J. Phys. Chem. B 105, 2264 (2001), and references therein.

[3] C. Voisin et al., Phys. Rev. Lett. 85, 2200 (2000).

[4] J. H. Hodak, A. Henglein, and G. V. Hartland, J. Chem. Phys. 112, 5942 (2000).

[5] M. Nisoli et al., Phys. Rev. Lett. 78, 3575 (1997).

[6] Y. Hamanaka et al., Phys. Rev. B 63, 104302 (2001).

[7] B. A. Smith et al., Chem. Phys. Lett. 270, 139 (1997).

[8] V. Halté et al., Appl. Phys. Lett. 75, 3799 (1999).

[9] M. B. Mohamed et al., Chem. Phys. Lett. 343, 55 (2001).

[10] S. Link et al., J. Phys. Chem. B 106, 945 (2002).

[11] $\Delta T_{e}^{m}$ measures the energy injected by the pump pulse and is defined as the temperature rise of a thermalized electron gas for the same energy increase.

[12] C. K. Sun et al., Phys. Rev. B 50, 15337 (1994).

[13] N. Del Fatti et al., Phys. Rev. B 61, 16956 (2000).

[14] R. Groeneveld, R. Sprik, and A. Lagendijk, Phys. Rev. B 51, 11433 (1995).

[15] M. I. Kaganov, I. M. Lifshitz, and L.V. Tanatarov, Zh. Eksp. Teor. Fiz. 31, 232 (1956) [Sov. Phys. JETP 4, 173 (1957)].

[16] K. Uchida et al., J. Opt. Soc. Am. B 11, 1236 (1994).

[17] B. Palpant et al., Phys. Rev. B 57, 1963 (1998).

[18] A. Taleb, C. Petit, and M. P. Pileni, Chem. Mater. 9, 950 (1997).

[19] M. Treguer et al., J. Phys. Chem. B 102, 4310 (1998).

[20] Isotropic free-electron band, phonon Debye model (there is no optical phonon), and static limit of the Lindhard's dielectric function for small momentum exchanges.

[21] W. Ekardt, Phys. Rev. B 29, 1558 (1984).

[22] A. Liebsch, Phys. Rev. B 48, 11317 (1993).

[23] J. Lermé et al., Phys. Rev. Lett. 80, 5105 (1998).

[24] A. Kara and T. S. Rahman, Phys. Rev. Lett. 81, 1453 (1998).

[25] C. S. Jayanthi et al., Phys. Rev. Lett. 59, 795 (1987).

[26] A. Eiguren et al., Phys. Rev. Lett. 88, 066805 (2002).

[27] C. Lopez-Bastidas, Phys. Rev. B 65, 035417 (2001).

[28] E. D. Belotskii and P. M. Tomchuk, Int. J. Electron. 73, 955 (1992). 